\title{
LA AGENDA OCULTA DE LA IGUALDAD DE GÉNERO \\ EN EL DESARROLLO
}

\author{
Juan David Gómez-Quintero* \\ Juan Agustín Franco Martínez **
}

\begin{abstract}
Resumen: Este artículo analiza los procesos de implantación de la igualdad de género en la Cooperación Internacional para el Desarrollo como expresión de la agenda oculta de la colonialidad. La hipótesis plantea que en el trasfondo de estos procesos se encierra un anhelo homogenizador por el cual las metas de Occidente se postulan como las metas del mundo. El objetivo es presentar un panorama general de la discusión teórica a partir de la revisión de las teorías críticas del feminismo moderno, del postcolonialismo y de algunos hallazgos de la antropología feminista. Las principales conclusiones plantean que los procesos de inclusión de la igualdad de género en el desarrollo son una forma de institucionalizar subrepticiamente los principios del feminismo occidental moderno en detrimento de los feminismos subalternos.
\end{abstract}

Palabras clave. Cooperación para el desarrollo, feminismo, igualdad de género, postcolonialismo.

\section{INTRODUCCIÓN}

Desde la segunda mitad del siglo xx, concretamente durante la década de los sesenta, los programas de cooperación para el desarrollo (CD)

\footnotetext{
" Doctor en Sociología, Universidad de Zaragoza. Profesor de la Universidad de Zaragoza (España). Correo electrónico: jdgomez@unizar.es

** Doctor en Economía, Universidad de Córdoba. Profesor de la Universidad de Extremadura (España). Correo electrónico: franco@unex.es
} 
de los países del Norte, ${ }^{1}$ fundamentalmente europeos, incorporan principios, estrategias, planes y programas dirigidos a la eliminación de la discriminación que sufren las mujeres y, desde mediados de los noventa, a la búsqueda de la igualdad de género (IG). Sin embargo, los mecanismos empleados para conseguir tales objetivos se han introducido de tal forma que desvirtúan la naturaleza del término "cooperación" y cuestionan el sentido mismo de la igualdad.

El propósito de este artículo es mostrar, a través de la revisión bibliográfica y la discusión teórica, el despliegue de la colonialidad del ser y del saber (Quijano, 2000; Mignolo, 2003; Escobar, 2005; Maldonado-Torres, 2007) en los procesos de introducción del valor social y el derecho jurídico a la igualdad entre hombres y mujeres en las políticas de la $\mathrm{CD}$. Este proceso no es atribuible a una intencionalidad racional y consciente de la mayoría de los actores de la CD que pretenden ejercer su poder de forma manifiesta.

Asuntos como la implantación de la IG ponen en evidencia la universalización descendente e inconsciente de algunos principios que se consideran imperativos morales de carácter universal. Esta implantación es parte del largo y sutil proceso por el cual la colonialidad representa la cara oculta de la modernidad.

En este sentido hablamos de la existencia de una agenda oculta (Gómez-Quintero, 2008), no como una pretensión malévola ni elaborada para introducir subrepticiamente determinados temas o prácticas de la sociedad occidental en las sociedades periféricas, sino como un conjunto de aspectos de la vida social moderna que, por su relevancia, legitimación y estimación en Occidente, son transmitidas indirectamente a otros sujetos que no han compartido históricamente tal cosmovisión.

La agenda oculta de la $\mathrm{CD}$ es un conjunto de creencias y prácticas latentes transmitidas por parte de los actores de la cooperación que establecen objetivos y formas de hacer propias de las sociedades modernas. La agenda oculta se diferencia de la agenda oficial o formal

\footnotetext{
${ }^{1}$ Por brevedad omitimos los matices que tendría este concepto así como el de Sur. Definimos, por tanto, el Norte como el conjunto de países que pertenecen al Comité de Ayuda al Desarrollo (CAD) de la Organización para la Cooperación y el Desarrollo Económico (OCDE).
} 
porque no revela abiertamente sus pretensiones, precisamente porque es incapaz de reconocerlas. La agenda oficial incorpora, de modo manifiesto, los objetivos y acciones que materializan la IG.

Este artículo plantea la relación entre colonialidad, desarrollo e igualdad de género como un continuo histórico que reproduce en las sociedades postcoloniales los valores y creencias de la modernidad occidental. Estos valores y creencias son parte de la cosmovisión moderna que clasificó a los sujetos colonizados como alteridades del colonizador, alteridades silenciadas desde y por los discursos hegemónicos del poder-saber de la modernidad.

La literatura sobre CD sugirió, desde hace algún tiempo, que los cambios sociales inducidos por la cooperación en los países del Sur debían hacerse "desde abajo" y "desde adentro" (Stohr, 1983; Kabeer, 1998); no obstante, en este trabajo planteamos como primera hipótesis que el asunto de la IG en la CD se ha introducido "desde arriba" y "desde fuera" como ejercicio de la colonialidad.

La introducción de la IG en la CD ha supuesto la traslación de los resultados de las conquistas (legítimas e imprescindibles) del feminismo occidental ${ }^{2}$ durante los últimos 150 años en Europa y Norteamérica, pero no de los procesos sociohistóricos que permitieron su consecución en las estructuras sociales, jurídicas y económicas de estas sociedades durante el siglo xx.

El impacto de la inclusión forzada de la IG como un asunto prioritario, transversal e inminente de las agencias del desarrollo, de los organismos multilaterales y de los estados del Norte, ha tenido como resultado ciertos artificios jurídicos, políticos y socioculturales que no sólo impiden la materialización real de la IG en las sociedades del

\footnotetext{
2 Denominamos feminismo occidental al conjunto de corrientes que asumen como universales los valores y creencias surgidas en la modernidad europea. En especial incluimos al feminismo radical, liberal y socialista. El feminismo radical diferencia claramente los sexos - mujeres y hombres - como dos categorías que conforman dos grupos identificables y, en su interior, homogéneos. El liberal se basa en los principios del utilitarismo que anteponen la felicidad como un derecho individual y un fin en sí mismo. Defiende la existencia de un Estado liberal que evite el control social de las mujeres y es afín al capitalismo. Por el contrario, el feminismo socialista defiende a la colectividad antes que al individuo y vincula el capitalismo con el patriarcado.
} 
Sur, sino que desvirtúan su legitimidad social como valor y derecho supuestamente universal. El asunto de la IG no ha sido el resultado del consenso o acuerdo de dos partes iguales que cooperan.

\section{LA IG EN LA AGENDA OFICIAL DEL DESARROLLO}

La IG se introdujo en la agenda oficial del desarrollo gracias al cambio del paradigma Mujer en el Desarrollo (MED) en Género en el Desarrollo (GED). La Cuarta Conferencia Internacional de la Mujer de Beijing en 1995 fue el hito que marcó tal transición.

A más de quince años de la Conferencia, no cabe duda de que ha habido cambios importantes en la situación social y económica de las mujeres a nivel mundial. En este periodo aumentó el número de mujeres inscritas en la educación, disminuyó el riesgo de mortalidad materna y aumentó la presencia de la mujer en la vida pública (política, empleo, migraciones) (Molyneaux, 2005). No obstante, la institucionalización del género y el empoderamiento de las mujeres contenidas en la Declaración de Beijing y en la Plataforma de Acción de Beijing han tenido algunas dificultades considerables. Una de ellas ha sido la tecnificación, politización y la distancia existente entre la IG tal como aparece en la agenda del desarrollo y la forma como se materializa en la vida cotidiana de los receptores. Si bien es cierto que la participación de la sociedad civil se ha incrementado en las últimas décadas en espacios globales como las cumbres mundiales y las conferencias internacionales, en la práctica, la operacionalización de las grandes metas ha resultado ajena a la cotidianidad de las personas que son receptoras o beneficiarias de la ayuda al desarrollo.

Como han mostrado varios autores (Rist, 2002; Nieto Pereira, 2001; Escobar, 1996), muchos discursos supuestamente alternativos del desarrollo promueven posturas flexibles, respetuosas y culturalmente compatibles con las "gentes del Sur" y, sin embargo, sus prácticas, actitudes sobre el terreno e interrelaciones con los autóctonos, evidencian una contradicción clara de la que no son conscientes (la agenda oculta). Promueven el desarrollo desde abajo, pero exigen unilateralmente los temas transversales, fomentan la identificación endógena de las 
problemáticas, pero su saber experto basado en indicadores sociales, económicos y demográficos, determina las necesidades de la gente.

La capacidad financiera y la experiencia acumulada de las organizaciones y movimientos del Norte, con frecuencia

$[\mathrm{H}] \mathrm{a}$ definido agendas que son "exportadas" al Sur, priorizando temáticas globales que no siempre se encuentran en el horizonte cognitivo y de demandas locales y que con frecuencia responden a un tratamiento conceptual y metodológico occidental, sin mencionar las diferenciaciones que se establecen al seleccionar recipiendarios, socios o contrapartes, de acuerdo con el lenguaje de las diversas onGS y agencias de cooperación del Norte (Serbin, 2001: 72).

De forma similar los trabajos de Sonia Álvarez (2001 y 2003) han puesto de relieve los procesos de institucionalización de los movimientos feministas en América latina y el Caribe (ALC) que han sido domesticados por el Estado y las grandes organizaciones del desarrollo a través de la "oenegización" y la "tercerización"; todo ello para reducir su poder contestatario frente a las estructuras sociales. Molyneux (2003) también cuestionó la autonomía y capacidad de representación de los movimientos de mujeres en algunos países de Centroamérica.

Podemos afirmar, por tanto, que el espíritu de Beijing no se ha consolidado totalmente, ni siquiera en la propia normativa de la Unión Europea. Así, la Estrategia Europea sobre la IG en la Cooperación (Comisión Europea, 2007) adolece de cierto grado de imposición y de etnocentrismo occidental, especialmente en el modo de aplicar las medidas que propone y en las recomendaciones que sugiere. Así, esta estrategia subraya principalmente la importancia de las mujeres como beneficiarias (objeto pasivo) de las medidas de CD, en vez de considerarlas como protagonistas (sujeto activo). También establece que la dimensión de género debe integrarse en la elaboración de los presupuestos de los países receptores; e incide en un planteamiento institucional vertical, que prioriza a los gobiernos como contraparte, destacando la obligación que tienen los países receptores de adoptar estrategias nacionales 
de reducción de la pobreza, y no sólo de denunciar la falta de recursos financieros.

En España se reconoce oficialmente cómo la IG no se ha desarrollado plenamente en la cooperación española (MAEC, 2007). Las principales deficiencias pueden resumirse en dos. Por una parte, las dificultades para institucionalizar la transversalidad del enfoque de género en los programas, proyectos y políticas públicas en materia de cooperación (AECI/ISDEMU, 2004; AECI, 2006; Ortiz et al., 2006; MAE, 1998 y 2001). Por otra parte, la falta de incremento efectivo del presupuesto, pese a las buenas intenciones declaradas.

\section{LA COLONIALIDAD DEL SER Y DEL SABER: LA IG COMO VALOR UNIVERSAL}

\section{Antecedentes de la colonialidad}

Sería ingenuo suponer que las historias de las hegemonías y las relaciones de poder comienzan con el hecho histórico de la colonización. En la estructura social de cientos de culturas y sociedades precoloniales las mujeres han sido tratadas como seres inferiores. Desde las restricciones a su participación en la vida pública, pasando por los tabúes en torno a su conducta (durante la menstruación, el embarazo o después del parto), hasta las transacciones matrimoniales por compra de la esposa, son muchos los casos de dominación sobre las mujeres documentados por la antropología (Moore, 1991).

El trabajo antropológico en Chiapas de Freyermuth Enciso (2003), por ejemplo, mostró la tragedia de muchas mujeres indígenas que, sometidas por la obediencia y los prejuicios de la cultura indígena, sufrían la violencia material y simbólica de las tradiciones y encontraban la muerte en el embarazo o el parto.

No obstante, queremos situar el foco de análisis sobre los conceptos de colonialidad del ser y del saber, que remiten a procesos por los cuales se construyó y consolidó una forma de pensamiento hegemónico que se universalizó en las sociedades postcoloniales. Si bien reconocemos la existencia de una desigualdad de género precolonial, colonial y postcolonial, nuestro interés se centra en la última etapa. A través de 
la colonialidad del ser se construyó a los otros de la modernidad, que, según el periodo histórico, fueron definidos como salvajes, paganos, incivilizados, atrasados o subdesarrollados.

Esta clasificación de los otros se cultivó y perfeccionó a través de la colonialidad del saber, que tuvo como soportes sociohistóricos y epistemológicos a la Ilustración del siglo xvin y a las ciencias sociales de los siglos xix y xx. En consecuencia, conceptos como civilización, progreso y desarrollo fueron desde entonces los paradigmas que articularon la implantación del proyecto moderno en sociedades periféricas como alC (Castro-Gómez y Mendieta, 1998).

La universalidad de la filosofía iluminista se hizo pensando en un supuesto sujeto trascendental particular abstraído de su espacio, su tiempo, su etnia y sus relaciones sociales. Las ciencias humanas y sociales aceptaron de tal forma estos planteamientos que se consideraron durante siglos como una parte vertebral del acervo cultural de "La Humanidad". Tan solo en las últimas décadas, algunos autores críticos (Ribeiro, 1968; Castro-Gómez y Mendieta, 1998; Quijano, 2000; Santos, 2003; Lander, 2003; Mignolo, 2000 y 2003) han advertido que dicha universalidad fue una construcción temporal y espacialmente particular: "En palabras de Hegel, el Espíritu Universal es cristiano y germánico. De este modo, deja fuera lo hispánico, lo judío y, desde luego, lo maya, lo náhuatl, etcétera" (De la Garza, 2002: 10). Así mismo, también deja fuera a las mujeres y a las distintas expresiones de lo femenino. La filosofía moderna no ha pensado al sujeto desde su realidad inmediata, por el contrario, ha tachado de primitivas y salvajes algunas de las historias y experiencias sociales con las que rivalizaba. Más aún, las observaciones kantianas sobre la geografía moral de los grupos humanos conocidos, afirmaban que, de la variedad de razas,

[L]os indios americanos, los africanos y los hindúes aparecen como incapaces de madurez moral porque carecen de "talento", el cual es un "don" de la naturaleza [...]. La raza de negros es completamente lo contrario de la de los americanos: están llenos de afecto y pasión, son muy vivaces, conversadores y vanidosos. Pueden ser educados 
como sirvientes (esclavos) o sea que permiten ser entrenados (Kant citado en Chukwudi Eze, 2008: 49-50).

Esas afirmaciones antropológicas fueron hechas por los mismos filósofos que sentaron las bases de la racionalidad occidental moderna, que constituyó, a su vez, la matriz epistémica de las ciencias sociales de los siglos xix y xx. En consecuencia, la planificación del desarrollo económico y social realizado por las grandes agencias internacionales y organismos multilaterales durante el siglo xx ha estado contagiada por estructuras de pensamiento que hunden sus raíces en la colonialidad. Cabe destacar en esas estructuras la enorme influencia del evolucionismo social, el funcionalismo sociológico y la concepción teleológica de la historia.

Con esas estructuras de pensamiento es imposible conocer la realidad de las sociedades africanas, indias y latinoamericanas, porque los planteamientos que permiten comprender sus relaciones sociales, su historia y su cultura están condicionados por las premisas de una racionalidad supuestamente neutral, pero internamente racista, etnocéntrica y androcéntrica. Estas estructuras de pensamiento son la base de la colonialidad (Castro-Gómez y Mendieta, 1998; Mignolo, 2000 y 2003; Quijano, 2000).

Concebimos la colonialidad como un sistema ideológico que, apoyándose en algunas ciencias sociales como la historia, la antropología y la sociología, justificó la lógica de la dominación de unos pueblos sobre otros. Dominación que, en la mayoría de los casos, también se validó con la ayuda de razonamientos filosóficos y preceptos morales que exponían argumentos altruistas, caritativos o benéficos para ayudar al otro, primitivo o atrasado, a superar los distintos estados de inferioridad en los que se encontraba con respecto a "La Civilización".

Por lo tanto, la colonialidad se refiere a un patrón de poder que opera a través de la naturalización de jerarquías raciales, sociales y de género que posibilitan la reproducción de relaciones de dominación territoriales y epistémicas que no sólo garantizan la explotación por el capital de unos seres humanos por otros a escala mundial, sino que también subalternizan y obliteran los conocimientos, experiencias y 
formas de vida de quienes son así dominados y explotados (Quijano, 2000).

Hasta los primeros logros del feminismo en el siglo $\mathrm{xx}$, las mujeres eran objeto pasivo de dominación del sistema patriarcal de Occidente y resultaban invisibles para las estructuras del pensamiento de la modernidad. Su lucha permitió la inclusión gradual de algunas reivindicaciones feministas en las estructuras jurídicas, sociales, económicas y epistemológicas durante ese período. No obstante, las reivindicaciones del feminismo estuvieron influenciadas por un espacio, un tiempo y un sustrato socio-cultural específico: el de las mujeres blancas occidentales de clases medias (Suárez y Hernández, 2008).

De este modo, el feminismo occidental no ha sido inmune a la influencia sutil de la colonialidad, lo que le ha llevado a erigirse a sí mismo como portavoz de las mujeres del mundo. El feminismo occidental es, desde hace algunas décadas, el feminismo hegemónico; que "habla desde una estructura de poder, y ha logrado imponer su agenda política como la única agenda válida para la construcción de la equidad de género" (Suárez y Hernández, 2008: 15). Esta estructura se ha impuesto en la definición de lo que eran y son las mujeres (colonialidad del ser) y en la manera de conocer a las mujeres y al género (colonialidad del saber).

\section{La colonialidad del ser}

La colonialidad del ser es entendida como "la dimensión ontológica de la colonialidad, en ambos lados del encuentro [...] un exceso ontológico que ocurre cuando seres particulares se imponen sobre otros, y además encara críticamente la efectividad de los discursos con los cuales el otro responde a la supresión como resultado del encuentro" (Escobar, 2005: 35. Cursivas del autor).

Este discurso legitimó, por tanto, la construcción de una historia de la civilización como trayectoria que partía de un estado primitivo de naturaleza y culminaba en Europa. Esta centralidad histórica de Europa (eurocentrismo) ha distorsionado la representación del otro, pero sobre todo, ha alterado la autocomprensión de sí mismo. La sociedad 
moderna se convirtió, así, "no sólo en el modelo al que debe aspirar el resto de las sociedades, sino en el único futuro posible para todas las otras culturas o pueblos" (Castro-Gómez, 2003: 154).

Del mismo modo, el feminismo occidental-moderno se postuló como el único referente posible para la definición de "las mujeres" del mundo y para indicar el camino de su liberación (Mohanty, 1991 y 2003).

\section{La colonialidad del saber}

Por el carácter universal de la experiencia histórica europea, las formas de conocimiento desarrolladas para la comprensión de esa sociedad se convirtieron en las únicas formas válidas, objetivas, universales de conocimiento (Lander, 2003: 23). Así, se produjo también una colonización cultural y epistemológica que se concretó en la colonialidad del saber, la cual supuso la hegemonización de un sistema de representación y conocimiento de Europa y desde Europa. Por lo tanto, este dispositivo de saber/poder, una vez universalizado y naturalizado, subalternizó otras representaciones y saberes que quedaron relegados a simples objetos de conocimiento, silenciados y sin poder de enunciación. Esta elaboración teórica contribuyó a la universalización de la ideología de la colonización y a la naturalización de las relaciones de dominación entre europeos y no-europeos.

Así, la colonialidad como ideología ha servido igualmente para justificar los abusos de la imposición de un orden colonial. Además de la legitimación de la agresión, el pretexto de la modernización se erigió, tal como lo muestran las obras de Nisbet (1981), Sztompka (1993) y Rist (2002), en la nueva creencia colectiva que aceptaría el mito de la racionalidad moderna y evitaría el rechazo a las secuelas de la destrucción del orden social, temporal y espacial de las llamadas sociedades tradicionales.

Los sistemas de pensamiento y representación eurocéntricos se convirtieron en un proyecto propio de los estados poscoloniales. Sin embargo, la diversa realidad latinoamericana contrastó radicalmente con la artificialidad de un orden jurídico y social creado a imagen del prototipo de civilización europea, por lo que el resultado fue la 
continuidad soterrada de la organización social colonial basada en moldes socio-raciales.

Por ejemplo, la idea de igualdad promovida por los principios de la Revolución francesa fue muy difícil de asumir en las nuevas repúblicas, especialmente porque el orden poscolonial no significó una ruptura radical que asimilara o reconociera al otro interno. El odio y miedo a la plebe se dejó traslucir en el lenguaje y producción jurídica de las élites: "Las élites criollas despreciaron a las castas no sólo por representar lo autóctono o por el 'fastidio hacia lo rústico', sino porque ellas estaban gobernadas por los 'vicios', las 'bajas pasiones' y la 'ignorancia”' (Aguilera y Vega, 1998: 61. Comillas originales).

Es en ese proceso en el cual surge una doble conciencia criolla (Mignolo, 2003) a partir de la cual las élites criollas se esforzaron en tratar de ser americanos sin dejar de ser europeos marcando una diferencia sustancial con su otro interno (cualquiera que fuera el eufemismo): gentes ociosas, gentes desperdigadas por los montes, castas de la tierra (Aguilera y Vega, 1998: 62-63). Por ello podemos afirmar que la identidad latinoamericana es una identidad fracturada, un tipo de "esquizofrenia ontológica" que anhela ser lo que no es y reniega del pasado, de la mezcla y del origen que sí es.

\section{IGUALDAD DE GÉNERO, CRÍTICA FEMINISTA Y POSTCOLONIALIDAD}

La construcción de los estados poscoloniales latinoamericanos implicó el profundo anhelo por adoptar la forma de una sociedad moderna al estilo de los estados europeos, pero tal proceso se hizo artificialmente a través de la imitación de algunos referentes de las sociedades modernas como la igualdad de derechos y las libertadas cívicopolíticas. La grandilocuencia de esas metas democráticas no implicó la incorporación de los otros internos como ciudadanos del Estado, sino muchas veces significó su negación cuando no su transformación.

En el imaginario de la identidad latinoamericana residía la imagen del criollo blanco o, a lo sumo, del mestizo letrado, varón, católico, hispanoparlante o lusófono y urbanita, como expresiones tropicalizadas 
de la europeidad. Este imaginario condujo, por tanto, al consecuente rechazo de las identidades negras, indígenas y campesinas tradicionales.

En este contexto, el sistema de clasificación de etnia/género colonial empeoró aun más las condiciones de vida precoloniales de las mujeres indígenas, negras, iletradas y campesinas. Es innegable la doble discriminación bajo el paradigma de la colonialidad para la conjunción etnia/género. Si la condición racial, mestiza o étnica situaba a determinados sujetos en los niveles más bajos de la estratificación social de las sociedades coloniales y poscoloniales, la condición de "mujer" ahondaba aun más esta discriminación.

Podemos afirmar que, en el caso del género, hubo una doble renegación. Ésta consiste en "la producción de identidades discriminatorias que aseguren la identidad "pura' y original de la autoridad" (Bhabha, 2002: 140-141). El rechazo a la identidad negra, indígena y mestiza (pobre e iletrada) aseguraba la cercanía, por oposición, a la pureza de la identidad blanca europea.

Tal rechazo se ha producido en el seno de los movimientos feministas. Las luchas del feminismo hegemónico occidental han liberado sólo a unos tipos determinados de mujeres. Incluso, buena parte de ese feminismo ha sido cómplice de la renegación desde la profunda inconsciencia de la colonialidad del ser. Como prueba de ello cabe mencionar el racismo del feminismo hegemónico latinoamericano que ha dejado a los movimientos de mujeres negras e indígenas fuera de los encuentros feministas latinoamericanos entre 1981 y 2002 (Suárez y Hernández, 2008). A partir del siglo xxi se reconocen las nuevas identidades femeninas en ALC y el valor de su acción política (Lagarde, 2003).

A pesar del reconocimiento activista e intelectual, la situación de millones de mujeres indígenas, negras y campesinas en ALC es, en la actualidad, lamentable. En el subcontinente hay una población de 50 millones de indígenas y 120 millones de afrodescendientes (PIAD) que representan alrededor de 33\% de la población de la región. Al analizar las diferencias socioeconómicas de la población eurodescendiente (PED), por un lado, y de la PIAD, por el otro, se observa una brecha significativa. Los niveles de pobreza son notoriamente mayores en la PIAD que en la PED (PNUD, 2010). 
Entre estas poblaciones las mujeres ocupan la posición más baja y subordinada. Desde 2006 se elabora un informe mundial que mide la brecha de género atendiendo a cuatro dimensiones básicas: economía, educación, salud y política. En 2010, en promedio (de una muestra total de 134 países), las mayores desigualdades de género se observaron en las dimensiones económica y política. En general, los países latinoamericanos presentan bajos niveles de desigualdad de género en salud y de educación pero una amplia brecha en las otras dos dimensiones (World Economic Forum, 2010).

En este artículo no ponemos en duda la necesidad de reducir o eliminar estas desigualdades. Sin embargo, la forma en que el asunto de la IG se ha configurado como una prioridad del desarrollo sí debe discutirse. Más que el respaldo o acompañamiento a un proceso de emancipación endógena de las mujeres discriminadas del Sur, lo que dilucidamos en la agenda oculta de la CD es la transmisión de principios, valores y acciones propios del feminismo hegemónico como expresión de la modernidad occidental.

Los argumentos para respaldar tal aseveración los podemos encontrar en cuatro cuestiones fundamentales: a) la supuesta universalidad de la libertad sexual y reproductiva de las mujeres; b) la oposición entre la secularización del feminismo occidental y la confesionalidad de algunos feminismos subalternos; c) la distancia simbólica entre el individualismo moderno y el comunitarismo tradicional, y d) la incorporación de las mujeres al mercado de trabajo del sistema capitalista como un acto de "liberación femenina".

Algunos programas y planes de la agenda formal de la igualdad de género en la $\mathrm{CD}$ plantean las libertades sexuales y reproductivas como un asunto universal. Podemos citar, por ejemplo, el Programa Vita de Salud Sexual y Reproductiva de la Agencia Española de Cooperación Internacional (AECI, 2005), y la Guía sobre Género y Cooperación de Médicos del Mundo (Sojo et al., 2002). En estas guías puede detectarse la visión feminista occidental sobre la autonomía individual en materia sexual y reproductiva. Dicha universalidad fue cuestionada en la propia Cumbre de Beijing (Gómez-Quintero, 1996) y argumentada por feministas africanas y musulmanas. Cabe destacar a las exponentes del Motherism como Acholonu (1995), las portavoces 
del Womenism como Alice Walker (1983) y otras voces que provienen del feminismo musulmán como Mahmood (2008).

De otro lado, algunos trabajos empíricos antropológicos han evidenciado la heterogeneidad discursiva y práctica en torno a la libertad sexual y reproductiva de las mujeres. Hace varias décadas los trabajos etnográficos de Margaret Mead (1935) constataron la diversidad de actitudes sociales hacia la vida sexual y reproductiva de mujeres nativas de Nueva Guinea. En un trabajo empírico con mujeres Timbira de Brasil, María Elisa Ladeira (1993) constató que la función reproductiva de las mujeres no las relegaba a una posición de subordinación sino de complementariedad. Esta posición también fue descrita por el trabajo de Maruja Barrig (2001) con mujeres del Cuzco a partir de la noción de "incompletitud".

Durante las última décadas las políticas estatales de desarrollo en ALC en materia de salud materno infantil han puesto en evidencia la imposición de la lógica expansionista del feminismo moderno sobre las cosmovisiones locales: el caso del uso extensivo del biberón entre la PIAD y los campesinos pobres durante los años ochenta supuso la incorporación de un símbolo visible de modernidad a costa de una elevada mortalidad infantil (Small, 1999: 28; Contreras y Gracia, 2005: 266).

Además, y a pesar de coincidir con buena parte del diagnóstico del Programa de Naciones Unidas para el Desarrollo (PNUD, 2010) sobre el desarrollo humano, creemos que su enfoque adopta inconscientemente los valores hegemónicos occidentales en esta materia. El PNUD (2010: 70) destaca la necesidad de realizar esfuerzos en reducir el número de los embarazos adolescentes en las zonas rurales de ALC a través de la promoción de la educación. Sugiere que se deben incrementar "las oportunidades para que las adolescentes realicen actividades acordes con su edad y sus intereses" como si la maternidad fuese un accidente que lastra sus proyectos vitales individuales y como si hubiese un consenso mundial sobre el significado de las actividades adecuadas para todas las adolescentes del mundo. Aunque pueda ser inapropiado o desafortunado, para muchas adolescentes de ALC y África, la maternidad es el único proyecto al que pueden aspirar ante la inexistencia, infravaloración o imposibilidad de aspirar a otros proyectos vitales (educativos o productivos). 
El segundo de los argumentos que evidencia el particularismo del feminismo hegemónico que marca la agenda oculta de la ig es la cuestión religiosa. La idea de Dios y las actitudes confesionales están muy presentes en el discurso de las mujeres indígenas, afroamericanas y campesinas de América latina y África. En muchas de estas regiones algunos grupos de mujeres encuentran en lo sagrado un motivo para la cohesión social, un sentido para sus vidas e, incluso, una forma de resistencia cotidiana. En muchos países de ALC, la Iglesia católica y los movimientos religiosos han apoyado las luchas de los pobres contra las estructuras que les oprimen y han reivindicado los derechos concretos de las mujeres. En Brasil, Chile y El Salvador las protestas de la Iglesia católica contra los abusos a los derechos humanos han conllevado la vinculación y el respaldo de grupos feministas (Molynaux, 2005).

Por otro lado, pero siguiendo con el segundo argumento, el trabajo antropológico de Ioan Lewis (1971; citada en Moore, 1991) con pastores nómadas del norte de Somalia mostró cómo las mujeres utilizan la condición de posesas espirituales para ejercer un tipo de protesta sutil ante la dominación patriarcal. El trabajo de Mahmood (2008) sobre el movimiento de las mujeres de la mezquita evidencia que los objetivos del feminismo musulmán pueden ser contestatarios y religiosos simultáneamente. Por su parte, Acholonu (1995) señala que el feminismo africano no debe desligarse del binomio Dios-Naturaleza ya que es la base del orden natural de las cosas. Esta misma relación está presente en las reflexiones del ecofeminismo (Shiva, 1995 y 2008).

La distancia entre el individualismo moderno y el comunitarismo tradicional queda patente cuando se comparan los discursos en torno a los hijos, la familia y el grupo. Durante las décadas de 1980 y 1990, el feminismo latinoamericano de corte socialista (Barrett, 1980; Lagarde, 1996) había identificado a la familia en el centro de la opresión femenina. No obstante, cabe preguntarse hasta qué punto las nuevas identidades femeninas latinoamericanas (Lagarde, 2003), los feminismos africanos y algunas evidencias antropológicas cuestionan esa centralidad.

Para la socióloga nigeriana Oyèrònké Oyewùmí (2003), que realizó estudios de campo con la sociedad Yoruba, la maternidad sobrepasa la propia cuestión de género, ya que es previa a lo social, prenatal, postnatal 
y "de por vida". Por tanto, afirma, no existe ningún equivalente para tal institución, ni rol de género similar, en el caso de los varones. Desde su punto de vista la maternidad precede a todo lo demás y, por tanto, no se puede esencializar ni trivializar su significado para las mujeres africanas. Los escritos del feminismo africano de Steady (1981) definen el feminismo africano como un feminismo humanista, maternal y naturalista. Destaca la importancia de la infancia en África, reivindica el reconocimiento de los distintos tipos de maternidad y critica el individualismo moderno al que antepone la cooperación femenina.

En los estudios antropológicos de Barrig (2001) en Perú también se destacaba que la categoría "individuo" no es funcional en la comunidad, ya que la persona está subordinada al colectivo y a lo social, reconociendo que no es autosuficiente:

Y ahí, tanto el varón como la mujer juegan un papel importante pues sólo cuando hay una relación establecida de pareja se puede considerar que una persona ha llegado a un grado de madurez y de completitud, mientras no tienen pareja, sienten que tienen fuertes carencias, la pareja integra y hace una unidad. Se reconoce a la pareja como integrante de la comunidad y no al individuo (Barrig, 2001: 83).

Molineaux (2005) señaló, diez años después de Beijing, que el cambio social en materia de IG en el desarrollo no puede seguir una senda uniforme ni ofrecer garantías de que se obtengan los resultados esperados. La idea de desarrollo, agrega, nunca está simplemente relacionada con una sola versión de la modernidad.

El último de los argumentos que esgrimimos frente a la occidentalización latente en la agenda oculta de la igualdad de género es, quizá, el más polémico (Franco, 2010 y 2011). En el contexto del desarrollo cabe preguntarse si en el trasfondo de la expansión universal de la emancipación femenina existe una lógica igualmente expansiva del capitalismo que pretende liberar a los sujetos "atados" por la "tradición" y la "cultura" para ser incorporados al mercado de trabajo (Franco, 2008 y 2009). Así, hay autores como Castro-Gómez (2003) 
y Amparo (2006), que han señalado que el desarrollo modernizador en América latina también ha consistido en la proletarización y asalarización de aquellos colectivos que aún eran esquivos para el capitalismo. Por su parte, Shiva (1995 y 2008) y Mohanty (1991 y 2003) plantean que las formas de emancipación femenina de algunas mujeres del Sur no deben pasar necesariamente por la proletarización sino por la autosuficiencia comunitaria en la producción agrícola. Por último, Molineaux (2005) afirma que no siempre el desarrollo económico y social ha aumentado el ámbito de la libertad humana. Los efectos de la liberalización sobre el crecimiento económico han sido desalentadores y, por falta de prestaciones y redes de seguridad social efectivas, han expuesto a millones de personas a la pobreza y el desempleo.

\section{CONCLUSIONES Y REFLEXIONES FINALES}

De la revisión y análisis de la literatura se ha comprobado cómo es posible poner en cuestión los discursos de verdad que han trazado los principios que subyacen a la igualdad de género en la cooperación internacional al desarrollo. Se ha puesto de manifiesto que la pluralidad teórica del feminismo constata la pluralidad social y cultural de la realidad de quienes han sido definidas y representadas por un sujeto (varón) enunciador. Sin embargo, hemos observado, con la ayuda de los feminismos subalternos o periféricos y de la antropología feminista, cómo el discurso del feminismo occidental se erigió a sí mismo como portavoz de las mujeres del mundo orientando y dirigiendo a sus otras como si ellas fuesen sujetos incapaces de conducir sus propias vidas. La existencia de la agenda oculta de la ig en la cooperación al desarrollo es una prueba de ese proceso.

La construcción de la ig ha sido el resultado de importantes conquistas sociales de movimientos sociales como el feminismo en sus diversas expresiones políticas, históricas y teóricas. Estas conquistas fueron el resultado de conflictos sociales gestados desde la propia concienciación de dominación que experimentaron muchos grupos de mujeres. No obstante, cuando la CD convencional obliga a incluir la IG en las agendas, reproduce la relación de autoritarismo que 
muchas mujeres han experimentado durante siglos. El resultado de esta institucionalización de la IG en la cooperación es, en definitiva, el traslado de los resultados de las conquistas pero no de sus procesos sociohistóricos.

Esta crítica no desmerece los grandes logros obtenidos por las distintas olas del feminismo, pero sí advierte sobre el peligro de la reproducción de la desigualdad heredada en su momento por las mujeres, no en abstracto, sino por las mujeres blancas, modernas, seculares, de clase media y occidentales durante los siglos XIx y xx. Cabe recordar que esa lucha era contra un tipo de autoridad patriarcal que había definido, doblegado e inferiorizado a las mujeres como "los otros" de los hombres (Simone de Beauvoir). Ahora no se trata sólo de un sistema patriarcal que sigue ejerciendo poder sobre muchas mujeres, también vemos que hay un sistema ideológico que podríamos denominar de la igualación o del igualitarismo que ejerce un poder legitimado a través de la historia de la lucha social y política del feminismo hacia las otras mujeres de aquel sujeto histórico: son las otras de las otras, mujeres tradicionales, creyentes, mestizas, pertenecientes a minorías, pobres o que viven en el Sur.

La construcción social de la igualdad de género no puede seguir imitando artificialmente los valores de la modernidad occidental porque los cambios sociales no responderán adecuadamente a las demandas sociales, jurídicas, económicas y culturales de las mujeres de las sociedades periféricas. Desconocer algunos principios y valores como el comunitarismo, el arraigo al territorio y al sistema de creencias de los sujetos protagonistas, así como las contribuciones de los feminismos subalternos es un ejercicio de autoritarismo y violencia simbólica.

\section{Fuentes CONSULTADAS}

Acholonu, C. O. (1995), Motherism: The Afrocentric Alternative to Feminism, Owerri, Nigeria: Alliances for Africa (AFA) Publications. Agencia Española de Cooperación Internacional para el Desarrollo (AECID) (2005), Guía para programas y proyectos de salud sexual y reproductiva en África. Programa Vita, Madrid: AECID. 
- (2006), Diagnóstico rápido sobre la integración del enfoque de género en la Cooperación Oficial en América latina (2001-2005), Madrid: AECID.

aecid/Instituto Salvadoreño para el Desarrollo de la Mujer (isdemu) (2004), Documento línea de base. Programas AEci. Proyecto de fortalecimiento de la aplicación del enfoque de género en las políticas públicas, San Salvador: AECID/ISDEMU.

Aguilera, M., Vega, R. (1998), Ideal democrático y revuelta popular: bosquejo histórico de la mentalidad política popular en Colombia, 1781-1948, Bogotá: Instituto Sindical María Cano (ISMAC)/ Centro de Estudios de la Realidad Colombiana (CEREC).

Álvarez, S. (2001), "Third Sector, Third Way, a Second Look: Contemporary Latin American(ist) Debates Revisited", ponencia en el XXIII Congreso Internacional de Latin American Studies Association (LASA), septiembre 6-8, Washington, D. C.: Memorias del Congreso. (2003), Um outro mundo (também feminista...) é possível: construindo espaços transnacionais e alternativas globais a partir dos, en Estudos Feministas, vol. 11, núm. 2, julio-diciembre, Florianópolis, Brasil: Centro de Filosofia e Ciências Humanas/ Centro de Comunicação e Expressão, Universidad Federal de Santa Catarina, pp. 533-540.

Amparo, O. (2006), Las instancias rectoras de las politicas de mujer y género en el contexto de la modernización del Estado en América latina, Bogotá: Oficina Asesora de Política Pública de Mujer y Géneros del Gobierno de Bogotá.

Barrett, M. (1980), Women's Oppression Today, Londres: Verso.

BARrig, M. (2001), El mundo al revés: imágenes de la mujer indígena, Buenos Aires: Consejo Latinoamericano de Ciencias Sociales (CLACSO).

Bнавна, H. K. (2002), El lugar de la cultura, Buenos Aires: Manantial.

Castro-Gómez, S. (2003), "Ciencias sociales, violencia epistémica y el problema de la "invención del otro", en Edgardo Lander (ed.), La colonialidad del saber: eurocentrismo y ciencias sociales, perspectivas latinoamericanas, Buenos Aires: CLACSO, pp. 145161. 
- Mendieta, E. (1998), Teorías sin disciplina: latinoamericanismo, poscolonialidad y globalización en debate, México: Edición de Castro-Gómez y Mendieta.

Chukwudi Eze, E. (2008), "El color de la razón: la idea de 'raza' en la antropología de Kant", en Henry Pager, Santiago Castro-Gómez, Emmanuel Chukwudi Eze; Walter Mignolo (comps.), El color de la razón, Buenos Aires: Del Signo, pp. 21-81.

Comisión Europea (2007), "Igualdad de género y capacitación de las mujeres en la cooperación al desarrollo CD". Comunicación de la Comisión al Parlamento Europeo y al Consejo "COM (2007) 100 final", Bruselas: Oficina de Publicaciones de la Comisión Europea.

Contreras, J., Gracia, M. (2005), Alimentación y cultura. Perspectivas antropológicas, Barcelona: Ariel.

De la Garza, M. T. (2002), Politica de la memoria. Una mirada sobre Occidente desde el margen, Barcelona: Anthropos.

Escobar, A. (1996), La invención del Tercer Mundo. Construcción y deconstrucción del desarrollo, Bogotá: Norma.

Franco, J. A. (2011), "La Renta Básica y la tragedia de la propiedad privada", en Cuadernos de Renta Básica, núm. 10, Madrid: Fim (Fundación de Investigaciones Marxistas) (en prensa).

(2010), "Hacia un modelo económico transhistórico", en Journal of Transpersonal Research, vol. 2, Wittnau, AlemaniaMadrid: European Transpersonal Association (EUROTAS)/Centre for a Consciousness' Psychology (Oxigeme), pp. 73-96. (2009a), "El humor en la pedagogía de las ciencias económicas", en Campo Abierto, vol. 28, núm. 1, Badajoz: Facultad de Educación-Universidad de Extremadura, pp. 53-67.

(2009b), "El proyecto pedagógico de la ONG "Basida", en Tendencias Pedagógicas, núm. 14, Madrid: Departamento de Didáctica y Teoría de la Educación-Universidad Autónoma de Madrid, pp. 353-362.

(2008), "Reflexiones sobre Renta Básica, productividad y conocimiento", Cuadernos de Renta Básica, núm. 9, Madrid: FIM, pp. 29-36. 
Freyermuth Enciso, G. (2003), Las mujeres de humo. Morir en Chenalhó: Género, etnia y generación, factores constitutivos del riesgo en la maternidad, México: Centro de Investigaciones y Estudios Superiores en Antropología Social (CIESAS).

Gómez-Quintero, J. D. (2008), Esbozos de la ambigüedad del proyecto moderno: el caso de la cooperación no gubernamental al desarrollo hispano-colombiana, tesis doctoral inédita, Zaragoza: Departamento de Psicología y Sociología-Universidad de Zaragoza.

(1996), "Un aspecto para tener en cuenta en la Conferencia Internacional de la Mujer", en Revista Universidad de San Buenaventura, núm. 5, junio, Medellín: Universidad de San Buenaventura, pp. 45-52.

Kabeer, N. (1998), Realidades trastocadas: las jerarquías del género en el pensamiento del desarrollo, México: Paidós.

Ladeira, M. E. (1993), "Las mujeres Timbira: control del cuerpo y reproducción social", en Soledad González Montes (coord.), Mujeres y relaciones de género en la antropología latinoamericana, México: El Colegio de México (colmex), pp. 105-139.

Lagarde, M. (2003), "De la igualdad formal a la diversidad: una perspectiva étnica latinoamericana", en Anales de la Cátedra Francisco Suárez, núm. 37, pp. 57-80.

(1996), Género y feminismo: desarrollo humano y democracia, Madrid: horas y HORAS, D. L.

LANDER, E. (2003), "Ciencias sociales: saberes coloniales y eurocéntricos", en Edgardo Lander (ed.), La colonialidad del saber: eurocentrismo y ciencias sociales, perspectivas latinoamericanas, Buenos Aires: CLACSO, pp. 11-40.

Lewis, I. (1971), Ecstatic Religion, Harmondsworth, uk: Penguin.

Ministerio de Asuntos Exteriores (mae) (2001), Metodología de evaluación de la cooperación española II, Madrid: MAE.

(1998), Metodología de evaluación de la cooperación española, Madrid: Cyan, Proyectos y Producciones Editoriales.

Ministerio de Asuntos Exteriores y de Cooperación (maec) (2007), Estrategia de "género en desarrollo" de la cooperación española, Madrid: MAEC. 
Mahmood, S. (2008), "Teoría feminista y agente social dócil: algunas reflexiones sobre el renacimiento islámico en Egipto", en Liliana Suárez Navas, Rosalva Aída Hernández Castillo (eds.), Descolonizando el feminismo. Teorías y prácticas desde los márgenes, Madrid: Cátedra/Instituto de la Mujer, pp. 165-221.

Maldonado-Torres, N. (2007), "Sobre la colonialidad del ser: contribuciones al desarrollo de un concepto", en Santiago CastroGómez, Ramón Grosfoguel (eds.), El giro decolonial. Reflexiones para una diversidad epistémica más allá del capitalismo global, Bogotá: Instituto de Estudios Sociales Contemporáneos (IESCO)Universidad Central/Instituto de Estudios Sociales y Culturales, Pensar-Pontificia Universidad Javeriana/Siglo del Hombre, pp. 127-167.

Mead, M. (1935), Sex and Temperament in Three Primitive Societies, Nueva York: Laurel.

Mignolo, W. (2000), "La colonialidad a lo largo y a lo ancho: el hemisferio occidental en el horizonte colonial de la modernidad", en Edgardo Lander (comp.), La colonialidad del saber: eurocentrismo y ciencias sociales. Perspectivas latinoamericanas, Buenos Aires: CLACSO, pp.55-85.

(2003), Historias locales/diseños globales, Madrid: Akal.

Mohanty, C. (2003), Feminism without Borders: Decolonizing Theory, Practicing Solidarity, Durham, NC: Duke University Press.

(1991), Third World Women and the Politics of Feminism, Bloomington, in: Indiana University Press.

Molyneaux, M. (2003), Movimientos de mujeres en América latina: estudio teórico comparado, Valencia: Universidad de Valencia/Instituto de la Mujer.

(2005) Igualdad de género: La lucha por la justicia en un mundo desigual, Génova: United Nations Research Institute for Social Deveolpment (UNRISD).

Moore, H. L. (1991), Antropología y feminismo, Madrid: Cátedra.

Nieto Pereira, L. (2001), Cooperación para el desarrollo y ong. Una visión crítica, Madrid: La Catarata.

Nisbet, R. (1981), Historia de la idea de progreso, Madrid: Fondo de Cultura Económica (FCE). 
Ortiz, A., Pineda, A. C., Prieto, P. (eds.) (2006), Políticas públicas de mujer y género en el nivel local en la Región Andina y España, Bogotá: Oficina Asesora de Política Pública de Mujer y Géneros. Oyewumi, O. (2003), "Abiyamo: Theorizing African Motherhood", Jenda. Journal of Culture and African Women Studies, núm. 4, la Parte, Revista en línea: Africa Resource Center, Inc. Artículo en línea disponible en: http://www.africaknowledgeproject.org/index. php/jenda/article/view/79. 10 de diciembre de 2010.

Pereira Gamba, F. (1902), La riqueza mineral de la República de Colombia, Bogotá: Imprenta de La Crónica.

Programa de Naciones Unidas para el Desarrollo (pnud) (2010), Informe Regional sobre Desarrollo Humano para América latina y el Caribe 2010, San José de Costa Rica: PNud.

Quijano, A. (2000), "Colonialidad del poder, eurocentrismo y América latina", en Edgardo Lander (comp.) La colonialidad del saber: eurocentrismo y ciencias sociales. Perspectivas latinoamericanas, Buenos Aires: Clacso, pp.201-246.

Ribeiro, D. (1968), Las Américas y la civilización. Proceso de formación y causas del desarrollo desigual de los pueblos americanos, Caracas: Biblioteca Ayacucho.

Rist, G. (2002), El desarrollo: historia de una creencia occidental, Madrid: La Catarata.

Serbin, A. (2001), "Globalifóbicos vs. globalitarios: Fortalezas y debilidades de una sociedad civil regional emergente", en Nueva Sociedad, núm. 176, noviembre-diciembre, Buenos Aires: Fundación Foro Nueva Sociedad/Friedrich Ebert Stiftung, pp. 67-86.

Shiva, V. (1995), Abrazar la vida. Mujer, ecología y desarrollo, Madrid: horas Y HORAS. (2008), Los monocultivos de la mente, Monterrey: Fineo.

Small, M. F. (1999), Nuestros hijos y nosotros, Buenos Aires: Grupo Zeta. Sojo, D., Sierra, B., López, I. (2002), Salud y género. Guía práctica para profesionales de la cooperación, Madrid: Médicos del Mundo.

Steady, F. C. (1981), The Black Woman Cross-Culturally, Cambridge: Schenkman Publishing Co. 
StöHr, W. (1983), Desarrollo desde abajo: el paradigma de desarrollo de abajo hacia arriba, y de la periferia hacia adentro, Programa de Capacitación, Comisión Económica para América Latina (CEPAL)/Instituto Latinoamericano y del Caribe de Planificación Económica y Social (ILPES), Documento CPRD-D/80.

Suárez Navas, L., Hernández Castillo, R. A. (eds.) (2008), Descolonizando el feminismo. Teorías y prácticas desde los márgenes, Madrid: Cátedra/Instituto de la Mujer.

Sztompka, P. (1993), La sociología del cambio social, Madrid: Alianza.

Walker, A. (1983), In Search of Our Mothers' Gardens, Nueva York: Harvest Books.

World ECONOMic Forum (2010), The Global Gender Gap Report 2010, Ginebra: World Economic Forum.

Fecha de recepción: 4 de enero de 2011 Fecha de aprobación: 3 de mayo de 2011 